\title{
Applicability of Bottom-View Cameras for Evaluation of Tablet Performance During Dissolution Testing
}

Tjaša Felicijan, Ana Krese, Aleš Mrhar, and Marija Bogataj*

Faculty of Pharmacy, University of Ljubljana, Aškerčeva 7, Ljubljana, SI-1000 Ljubljana, Slovenia

\section{ABSTRACT}

Visualization techniques are frequently used to provide additional information on dissolution processes. The camera system used here, placed underneath the dissolution bath, enabled a bottom view into each separate vessel with the possibility for continuous video recording throughout dissolution testing. Drug release and swelling of hydroxypropyl methylcellulose (HPMC) matrix tablets containing either diclofenac sodium or paracetamol were simultaneously evaluated in a series of media with pH values of 3.0 to 7.5. Swelling of HPMC tablets was studied using two methods: evaluation of the relative change in tablet volume using the camera system and evaluation of relative change in tablet mass based on tablet weighing. The correlation between evaluated parameters in the two methods was high at all $\mathrm{pH}$ values for the tablets studied. Swelling of diclofenac sodium tablets was lowest at $\mathrm{pH} 3$, highest at $\mathrm{pH} 4$, and then decreased with the increasing $\mathrm{pH}$ of the medium. In contrast to the swelling results, drug release from diclofenac sodium tablets increased with increasing pH of the medium. At lower media $\mathrm{pH}$ values (i.e., 3.0, 4.0, and 5.0), the surface of the swelled layer appeared completely white and nontransparent, whereas at higher $\mathrm{pH}$ values, a transparent gel layer was clearly seen using the camera system. It is presumed that because of its low solubility, diclofenac precipitated as an acid on the tablet surface at lower $\mathrm{pH}$ values and was seen as a white swelled layer. For paracetamol tablets, drug release and swelling were $\mathrm{pH}$ independent, and a gel layer was clearly seen in all media tested (pH 3.0, 5.0, and 7.0). Thus, for HPMC tablets containing diclofenac sodium, the use of cameras provided additional information on the drug release and swelling process.

KEYWORDS: Evaluation of swelling; bottom-view cameras; dissolution; HPMC; diclofenac sodium.

\section{INTRODUCTION}

$\mathrm{V}$ isualization techniques are important tools in the observation of different processes during dissolution testing. Various approaches have been used for studying tablet behavior during the dissolution process. For example, Kazarian and van der Weerd (1) used simultaneous macro photography and Fourier transform infrared spectroscopy-attenuated total reflectance (FTIR-ATR) imaging to interpret physical changes of hydroxypropyl methylcellulose (HPMC) and buflomedil pyridoxal phosphate tablets during dissolution in a specially designed cell. Using macro photography alone, changes in tablet appearance were observed, but a quantification of the substance concentration was not possible. However, with concomitant use of FTIR-ATR imaging, both water ingression and drug concentrations at various locations in the tablet were quantified, showing the relationship between visually observed fronts (i.e., gel formation front, erosion front) and different amounts of water. Morita et al. (2) also used a camera to analyze the disintegration time of rapidly disintegrating tablets. Tres et al. (3) used photographic images during dissolution in a specially designed flow cell to observe tablets containing

*Corresponding author.

Dissolution

Technologies NOVEMBER 2016 differing amounts of felodipine, a drug with low solubility in water. Dissolution was complete for tablets containing smaller amounts of the drug, but for formulations with higher drug content, the initial swelling was followed by a decrease in tablet size, although the formulations had the same appearance before the dissolution process. Cao et al. (4) studied photo images of tablets coated with HPMC as a function of time during release studies in a USP paddle apparatus with a camera at the side of the dissolution vessel. Photo images of the initial swelling and subsequent disintegration of the coated tablet related well to the release of the drug. Tieu et al. (5) used cameras providing side and bottom views into the dissolution vessel to assess different potentials for monitoring the dissolution process via video and concluded that cameras provided an easy assessment of the presence or absence of the tablet and its placement in the dissolution vessel. They also proposed the use of cameras during formulation development and studied camera recordings of the dissolution system with different vessels and vessel heating types, as well as different camera angles to assess the use of video monitoring in several types of equipment. 
HPMC matrix tablets for oral use are designed to control drug release while passing through the gastrointestinal tract. When in contact with aqueous media, hydrophilic HPMC matrices become hydrated, and the formation of the gel layer (so-called rubbery polymer) on the matrix surface is due to the entry of water and the relaxation of polymer chains. In the gel layer zone, water entry, drug dissolution, and diffusion take place together with matrix erosion (6-8). Three fronts have been defined in the hydrophilic matrix system (9). The swelling front separates the dry tablet core from the gel layer; here, the polymer transforms from a glassy to a rubbery state. The erosion front represents the boundary between the dissolution medium and the swelled matrix. Inside the gel layer, the diffusion front separates the solid drug from the dissolved drug. The thickness of the gel layer might be of great importance in drug release, especially the gel layer between diffusion and the erosion front $(10,11)$.

The process of drug release is governed by two mechanisms: the diffusion of dissolved drug through the gel layer and erosion of the swelled polymer matrix surface exposing space for drug release. These mechanisms usually occur simultaneously $(7,11,12)$. Drug solubility affects gel characteristics, and drug release and poorly soluble drugs may reduce entanglement of the polymer chains and lower the gel strength $(13,14)$. Thus, erosion is the dominant release mechanism. For highly soluble drugs, release from the matrix is supposed to be governed mainly by diffusion. Moreover, highly soluble drugs might act as pore formers in the gel and enhance water penetration or act as additional osmotic contributors and increase the growth of the gel layer (15).

The assessment of the influence of individual drugs on release from the matrix is on a case-by-case basis. Apart from solubility, other drug characteristics such as molecular weight, chirality, particle size, and the $\mathrm{HPMC} /$ drug ratio in the matrix could affect release from the matrices (16). Additionally, the weakening or strengthening of the gel can be observed with various drugs, based on the disruption of the gel layer or altering water binding to the polymer (6).

The purpose of the present study was to assess the use of cameras placed underneath the dissolution bath to gain insight into dosage form performance during the dissolution process. Tablet swelling is a common approach for controlling drug release and can be easily observed. Therefore, swelling was chosen as a study parameter for assessing the applicability of the camera system. HPMC matrix tablets containing either diclofenac sodium (DF-Na) or paracetamol were studied, and tablet swelling was evaluated using a camera and compared with tablet weighing.

\section{MATERIALS AND METHODS}

\section{Materials}

DF-Na and paracetamol were purchased from SigmaAldrich (USA). HPMC (Metolose 90SH-4000SR) was manufactured by Shin-Etsu Chemical CO (Japan) and kindly donated by Harke Pharma (Germany). Citric acid monohydrate $\left(\mathrm{C}_{6} \mathrm{H}_{8} \mathrm{O}_{7} \cdot \mathrm{H}_{2} \mathrm{O}\right)$ and disodium hydrogen phosphate $\left(\mathrm{Na}_{2} \mathrm{HPO}_{4}\right)$ were purchased from Merck KGaA (Germany). Titrisol sodium hydroxide concentrate and Titrisol hydrochloric acid concentrate (Merck KGaA, Germany) were used for the preparation of $1 \mathrm{M}$ sodium hydroxide $(\mathrm{NaOH})$ and $1 \mathrm{M}$ hydrochloric acid $(\mathrm{HCl})$, respectively.

\section{Tablet Manufacture}

Tablets containing HPMC and either DF-Na or paracetamol ( $25 \%$ or $50 \%$ ) were prepared at the Faculty of Pharmacy, University of Ljubljana, Slovenia. The components were mixed and compressed manually using a Kilian SP300 single-punch tablet press (IMA Kilian, Germany) with 12-mm flat-faced punches. Tablet mass was set at approximately $400 \mathrm{mg}$.

\section{Dissolution Studies}

An Agilent 708-DS (Agilent Technologies, USA) USP paddle dissolution apparatus was used for dissolution testing.

The media were Mcllvaine buffers with $\mathrm{pH}$ values from 3.0 to 7.5 that were diluted 4-fold. Mcllvaine buffers are mixtures of two stock solutions, $0.2 \mathrm{M} \mathrm{Na}_{2} \mathrm{HPO}_{4}$ and 0.1 $\mathrm{M}$ citric acid, in different volumetric ratios to achieve the desired $\mathrm{pH}$ value ( $\mathrm{pH}$ range of $2.2-8)$. Specially designed stainless-steel wire sinkers were used.

Degassed dissolution medium $(900 \mathrm{~mL})$ was placed in the dissolution vessel and maintained at $37 \pm 0.5^{\circ} \mathrm{C}$. The agitation rate was set at $75 \mathrm{rpm}$. To determine the fraction of drug released, 5-mL samples were collected manually at predetermined time points $(0,30,60,120,180$, and 240 $\mathrm{min}$ ) and filtered through $0.45-\mu \mathrm{m}$ regenerated cellulose membrane filters (Sartorius Minisart RC, Sartorius, Germany); the first $2 \mathrm{~mL}$ of filtrate was discarded. Drug concentration in the samples was measured in an Agilent 8453 UV-vis spectrophotometer (Agilent Technologies, USA) at $276 \mathrm{~nm}$ for DF-Na and $243 \mathrm{~nm}$ for paracetamol. At each sampling point, a short video was recorded using the camera system before the samples were collected. Next, tablets were taken out of the vessels and weighed. 
To control the position of the tablet in the vessel before and after weighing, photographs were also taken shortly after the tablets were transferred back to the dissolution vessels.

\section{Evaluation of Swelling by Tablet Weighing}

At each time point, paddle stirring was temporarily stopped; tablets were taken out of the vessels and gently wiped with a paper cloth, then weighed with the sinkers. The weight of the sinker was subtracted from the weighed mass to determine the mass of the tablet at each time point $\left(m_{t}\right)$. As soon as weighing was finished, the tablets were transferred back into the vessels and dissolution was continued. The relative change in tablet mass at each time point $\left(m_{[\text {rel] } t}\right)$ was calculated using:

$$
m_{[r e l] t}=\frac{m_{t}}{m_{0}}
$$

where $m_{t}$ is the mass of the swelled tablet at a particular time point and $m_{0}$ is the dry tablet mass at the beginning of the experiment.

\section{Evaluation of Swelling Using a Camera System}

A dissoGUARD PRO dissolution surveillance system (Merel d.o.o., Slovenia) placed underneath the dissolution bath was used for visual observation of the tablets during dissolution (Figure 1A,B). Due to the placement of separate cameras in the dissolution surveillance system precisely beneath each dissolution vessel, an individual view into the vessel from the bottom is possible (i.e., bottom-view cameras). The system comprises cameras with highquality optics beneath the vessels (Sony 1/4" CCD image sensor with $\mathrm{M} 12 \times 0.5$ adjustable lens; resolution $640 \times$ 480 pixels) and additional flexible side camera (resolution HD $1920 \times 1080$ pixels). The camera system software (Merel dissoGUARD PRO version V1.1.13) enables

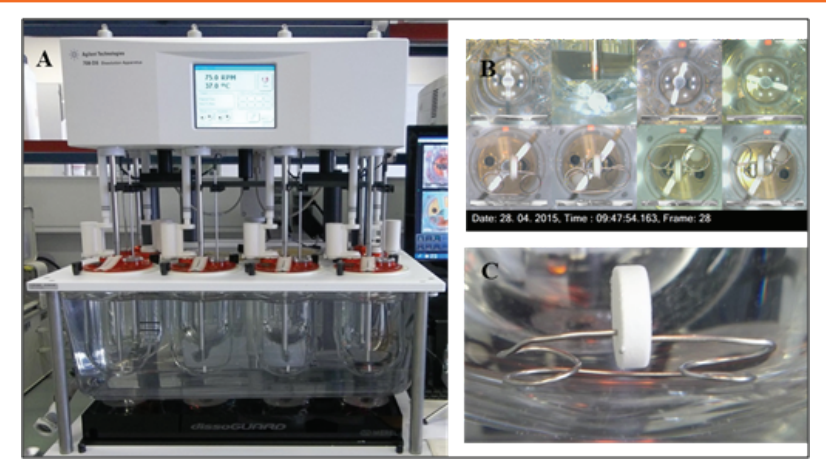

Figure 1. (A) Dissolution system with cameras placed underneath the dissolution bath. (B) View into dissolution vessels obtained by cameras. (C) Specially designed sinker enabling a strictly vertical position of the tablet. prediction and measurement of different parameters of the dissolution system by specific algorithms. For glare reduction, the intensity of the white illumination was turned off completely, although the intensity could be set to a minimum setting. This function can also provide red light instead of white light to protect light-sensitive drugs from degradation (17).

A 10 -sec video was recorded at each sampling point and shortly after tablet placement back into dissolution vessels after tablet weighing using the dissoGUARD PRO with images captured at $100-\mathrm{ms}$ intervals. A photograph where tablets were best seen was saved from each video. Relative change in tablet volume at each sampling time point $\left(V_{[\text {rel]t }}\right)$ was calculated using:

$$
V_{[\mathrm{rel}] t}=\frac{V_{t}}{V_{0}}
$$

where $V_{t}$ is the calculated tablet volume at time $t$ and $V_{0}$ is the calculated tablet volume at time point zero in the dissolution vessel.

Both volumes were calculated according to the cylindrical volume equation:

$$
V=\pi \times r^{2} \times h
$$

where $V$ is the volume, $r$ is the radius, and $h$ is tablet thickness. For determination of $V_{t}$ and $V_{0}$, the tablet radius at a certain time point $\left(r_{t}\right.$ or $\left.r_{0}\right)$ and tablet thickness at a certain time point $\left(h_{t}\right.$ or $\left.h_{0}\right)$ were determined from images obtained by cameras using the Microsoft Paint program.

Because the light passed through multiple barriers between the tablet and the camera, the measured dimensions of the tablets are not true dimensions. However, because only relative values $\left(V_{[\text {[rel]t }}\right)$ were calculated, no corrections were made.

\section{RESULTS AND DISCUSSION}

In the present work, the swelling and dissolution of hydrophilic matrix tablets containing DF-Na or paracetamol were studied. A simple tablet composition of only two substances was chosen because the purpose was to study processes that take place during dissolution. HPMC was chosen as the matrix polymer, and swelling was expected to be the major process observed by two different methods during dissolution: evaluation of relative change in tablet mass and evaluation of relative change in tablet volume using a camera system. 


\section{Evaluation of Swelling Using a Camera System}

The cameras of the dissoGUARD PRO dissolution surveillance system used in this study primarily measure and evaluate various physical parameters, such as paddle or basket wobble, paddle or basket rotation speed, and proper centering of vessels and shafts (17). This system was chosen because it enables an upward view into the dissolution vessels and the distance between the camera and dissolution vessel is fixed. This distance is equal for each camera-vessel pair in the system. An additional flexible camera can provide a side view into any of the dissolution vessels. The system enables continuous video monitoring of the dissolution process or recordings of short videos, as used in this study, with different capturing time intervals and numbers of shot frames per second (up to 15 frames per second). During video recording, the system can also detect and mark certain errors such as the stopping of paddles or baskets. After the video is recorded, events such as tablet drop, basket immersion, or sampling can be marked. In the present study, this type of camera was used to follow tablet performance under different dissolution conditions.

The camera system has advantages and disadvantages in studying tablet swelling. Cameras placed underneath the dissolution bath do not interfere with the dissolution process, and tablet performance can be followed continuously. With the use of another computer program (Microsoft Paint), measuring the tablet dimensions from recorded photographs is also possible, as well as measuring gel layer thickness when it is clearly seen. However, cameras and the corresponding software are primarily developed to monitor the physical parameters of the dissolution system; therefore, determinations of tablet dimensions might show greater variability. In addition, it is difficult to determine true tablet and swelled-layer dimensions as the tablet is immersed in an aqueous medium in the dissolution vessel and the vessel itself is in a water bath; thus, the light has to pass through materials with different refractive indices before reaching the camera. During dissolution, values at each sampling time point were compared with values at time zero. Thus, all final results were interpreted in a relative sense, and differences in measured and real tablet dimensions were not relevant in this work. Also, when calculating $V_{t}$ and $V_{0}$, the cylindrical volume equation was used as an approximation of tablet shape, although they were sometimes concave after a certain time in the dissolution medium. This was manifested primarily in media with lower $\mathrm{pH}$ values.

The relative change in tablet volume $\left(V_{[r e l] t}\right)$ was calculated from tablet dimensions measured by cameras placed underneath the dissolution bath. For such determinations, the tablet had to be positioned strictly vertically. A specially shaped sinker was made from stainless-steel wire (Figure 1C). The tablet was inserted into the sinker between the two ends of the wire, which held the tablet vertical and elevated from the bottom of the vessel. With these sinkers, it was possible to observe the axial and radial dimensions of the tablet at the same time. Readings of both tablet diameter and thickness were necessary since tablet swelling was more extensive in the axial direction than in the radial direction, which is in accordance with Rajabi Siahboomi et al. (18). Throughout the experiment (and during the tablet weighing process) it was necessary to prevent translocation of the tablets (within the sinkers) closer to the bottom of the vessel or away from the strictly vertical position, as this could affect measurements.

\section{Evaluation of Swelling by Tablet Weighing}

To assess the applicability of the camera system, swelling results were compared with a second method, evaluation of relative change in tablet mass $\left(m_{[\text {rel] } t}\right)$ determined by tablet weighing during dissolution. The swelling process is frequently monitored by weighing the tablets during dissolution, where the swelling index (19) or relative swelling and dissolution medium uptake (20) can be calculated from the change in tablet mass. A disadvantage of this method is that the dissolution process has to be stopped so the tablets can be taken out of the vessels, drained, and weighed. In the present study, tablets were transferred back to vessels after weighing so that the dissolution could proceed; thus, the same tablets were weighed at all sampling time points. Determination of $m_{\text {[rel]t }}$ provided an easy assessment of tablet swelling despite some limitations of the method such as variability in tablet wiping (i.e., inadequate or excess wiping of remaining media droplets on sinker and tablet surface with possible injury or partial removal of the gel layer during wiping), interruption of the dissolution process at each time point, and variability in the time during which the tablet was not exposed to the medium while weighing.

Interruption of the dissolution process and tablet manipulation could influence the dissolution process; therefore, in subsequent experiments, tablets were weighed only at the beginning and end of the experiment. The relative differences in drug release between tests with and without weighing at all sampling points were up to $14 \%$ at higher media $\mathrm{pH}$ values. In media with lower $\mathrm{pH}$ values, there were greater differences (up to $40 \%$ ) from

Dissolution

NOVEMBER 2016 Technologies 
the much smaller fraction of drug released than at higher $\mathrm{pH}$ values. However, because the results were interpreted only in a relative sense (comparison of two methods for the evaluation of swelling profiles of the same tablets), the observed differences in drug release did not influence the conclusions.

A calculation of the relative change in tablet volume was not possible by weighing because of the different densities of the dry tablet core and swelled tablet matrix. Therefore, the relative change in table volume was not determined.

\section{Comparison of Tablet Swelling Results Obtained in the Two Methods}

The swelling of tablets containing HPMC and DF-Na was evaluated using cameras and compared with results obtained by tablet weighing. Figure 2 shows the comparison of the average $V_{\text {[rel]t }}$ profile determined using cameras and the average $m_{\text {[rel] } t}$ profile determined by weighing for tablets containing $50 \%$ DF-Na in $\mathrm{pH} 6.8$ medium. The increasing trend of both $V_{[\text {rel] } t}$ and $m_{[\text {rel] } t}$ increased with increasing time in the dissolution vessel can be observed. However, calculated values of $V_{[\text {[rel] } t}$ are larger than $m_{\text {[rel]t } t}$ and are probably due to experimental factors such as refraction of light during photography and other issues in both methods, as described earlier. In addition, variability in the determination of $V_{\text {[rel]t }}$ was slightly higher than in $m_{[r e l]}$, as shown by the standard deviations in Figure 2. High correlation between average $V_{\text {[rel] } t}$ and average $m_{\text {[rel] } t}$ for tablets containing $50 \%$ DF-Na in the medium at pH 6.8 is shown in Figure 3. When assessing the correlation of swelling results in the two methods at $\mathrm{pH} 6.8$, determination coefficients $\left(R^{2}\right)$ for each of the four parallel experiments $\left(R_{1}{ }^{2}=0.9841\right.$, $R_{2}{ }^{2}=0.9813, R_{3}{ }^{2}=0.9910$, and $R_{4}{ }^{2}=0.9899$ ) were lower than $R^{2}$ for the average results $\left(R^{2}=0.9927\right)$, although correlations remained high. Correlations of average $V_{\text {[rel] } t}$ and average $m_{\text {[rel]t }}$ were also high in other tested media for tablets containing $50 \%$ or $25 \%$ DF-Na or paracetamol (Table 1).

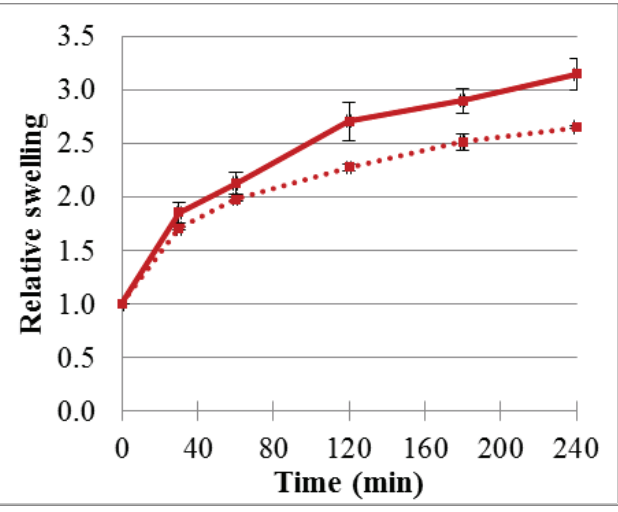

Figure 2. Relative swelling of tablets determined in two methods. The solid line represents $V_{\text {[rellt }}$ determined using cameras; the dotted line represents $m$, Tablets contained $50 \%$ DF- $N a$; medium $\mathrm{pH}$ was $6.8 ; n=4$ (values are mean $\pm S D$ ).

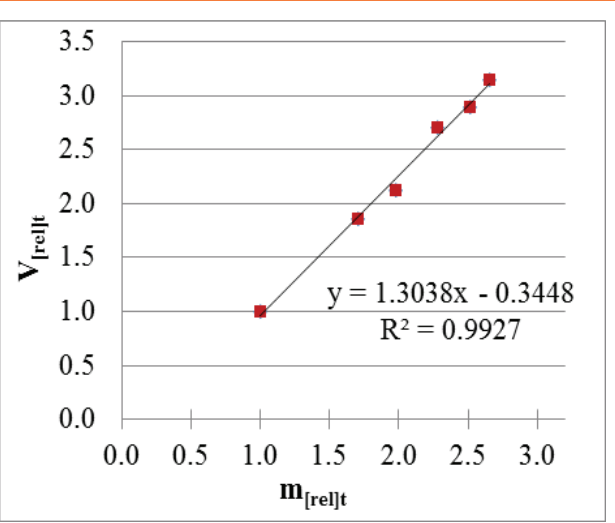

Figure 3. Correlation of average $V_{\text {frelt }}$ determined using cameras, and average $m_{\text {[rellt }}$ determined by weighing at different sampling points. Tablets contained 50\% DF-Na; medium pH was 6.8; equation of linear regression line and determination coefficient $\left(R^{2}\right)$ are shown $(n=4)$.

Table 1. Equations of Linear Regression Lines Through Average Points of $V_{\text {[rel]t }}(=y)$ and $m_{[r e l] t}(=x)$ Determined at Different Time Points, With Determination Coefficients $\left(R^{2}\right)$ for Different Tablets in Media with a Range of $\mathrm{pH}$ Values

\begin{tabular}{|c|c|c|c|c|c|c|c|c|}
\hline \multirow{3}{*}{ pH } & \multicolumn{8}{|c|}{ Tablet composition } \\
\hline & \multicolumn{2}{|l|}{$50 \% \mathrm{DF}-\mathrm{Na}$} & \multicolumn{2}{|l|}{$25 \% \mathrm{DF}-\mathrm{Na}$} & \multicolumn{2}{|c|}{$50 \%$ paracetamol } & \multicolumn{2}{|c|}{$25 \%$ paracetamol } \\
\hline & equation & $R^{2}$ & equation & $R^{2}$ & equation & $R^{2}$ & equation & $R^{2}$ \\
\hline 3.0 & $y=1.3779 x-0.3991$ & 0.9950 & $y=1.0875 x-0.1167$ & 0.9984 & $y=1.0814 x-0.0887$ & 0.9929 & $y=1.1041 x-0.1073$ & 0.9972 \\
\hline 4.0 & $y=1.247 x-0.2954$ & 0.9974 & $y=1.1151 x-0.1249$ & 0.9980 & & & & \\
\hline 5.0 & $y=1.1566 x-0.2145$ & 0.9871 & $y=1.1591 x-0.1341$ & 0.9966 & $y=1.1803 x-0.1911$ & 0.9910 & $y=0.9031 x-0.0167$ & 0.9979 \\
\hline 6.0 & $y=1.2122 x-0.2517$ & 0.9888 & $y=1.0675 x-0.0832$ & 0.9994 & & & & \\
\hline 6.8 & $y=1.3038 x-0.3448$ & 0.9927 & $y=1.0907 x-0.1023$ & 0.9982 & & & & \\
\hline 7.0 & $y=1.2307 x-0.2582$ & 0.9957 & $y=1.0639 x-0.1063$ & 0.9987 & $y=1.0092 x-0.0457$ & 0.9780 & $y=1.0287 x-0.063$ & 0.9917 \\
\hline 7.5 & $y=1.2379 x-0.2839$ & 0.9897 & $y=1.0658 x-0.0717$ & 0.9984 & & & & \\
\hline
\end{tabular}




\section{Influence of pH on Swelling and Drug Release from HPMC Matrices}

The influence of media $\mathrm{pH}$ (3.0-7.5) on DF-Na dissolution and tablet swelling was evaluated in diluted Mcllvaine buffers in both methods.

As has already been demonstrated (21), DF-Na dissolution is strongly dependent on the $\mathrm{pH}$ of the medium (Figure 4). The dissolution rate increased with increasing $\mathrm{pH}$, which was expected and is in accordance with the higher solubility of $\mathrm{DF}-\mathrm{Na}$ at increasing $\mathrm{pH}$ (22). The relative swelling of tablets containing $50 \%$ DF- $\mathrm{Na}$ in media at different $\mathrm{pH}$ values, determined in both methods, is shown in Figure 5. The relationship between swelling profiles in media with different $\mathrm{pH}$ values differs from the previously described relationship of dissolution profiles in the same media. The least amount of swelling was observed at $\mathrm{pH} 3$, the highest at $\mathrm{pH} 4$, and then swelling decreased with increasing media $\mathrm{pH}$ with no differences at the highest $\mathrm{pH}$ tested. The same dissolution and swelling trends were observed for tablets containing 25\% DF$\mathrm{Na}$. With increasing $\mathrm{pH}, \mathrm{DF}-\mathrm{Na}$ solubility increased and the amount of dissolved drug was consequently higher. At pH 3.0, DF-Na solubility was still very low $\left(\mathrm{p} K_{\mathrm{a}}=3.8\right)$ (23). It is assumed that because of the high drug-to-HPMC ratio and the low solubility of the drug, water transport into the matrix can be restricted, and therefore, swelling might also be limited. Increased swelling is indicated at $\mathrm{pH}$ 4.0, where the solubility of diclofenac supposedly hinders swelling less than at lower $\mathrm{pH}$ values. However, with increasing $\mathrm{pH}$, the volume of the swelled tablet is smaller than at $\mathrm{pH}$ 4.0. This might be a consequence of a large amount of dissolved DF-Na diffusing out of the matrix. In addition, high drug release supposedly contributes to disentanglement of polymer chains and erosion of the matrix.

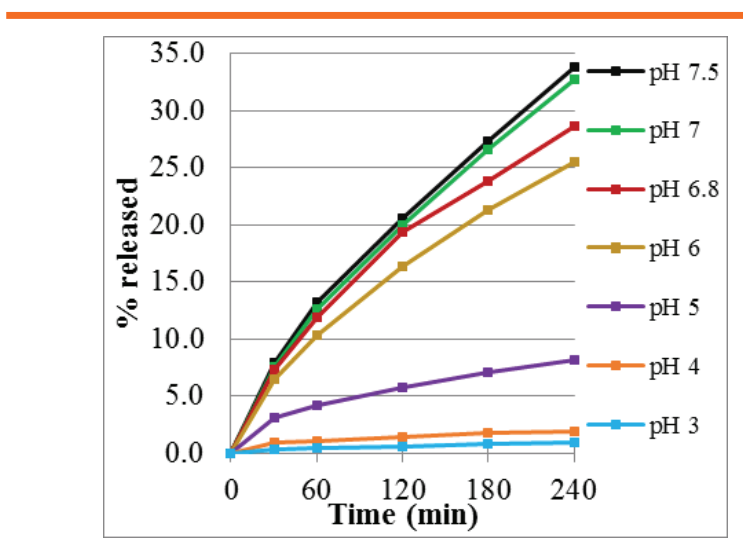

Figure 4. Dissolution profiles of tablets containing 50\% DF-Na in media with $\mathrm{pH}$ values from 3.0 to 7.5 .
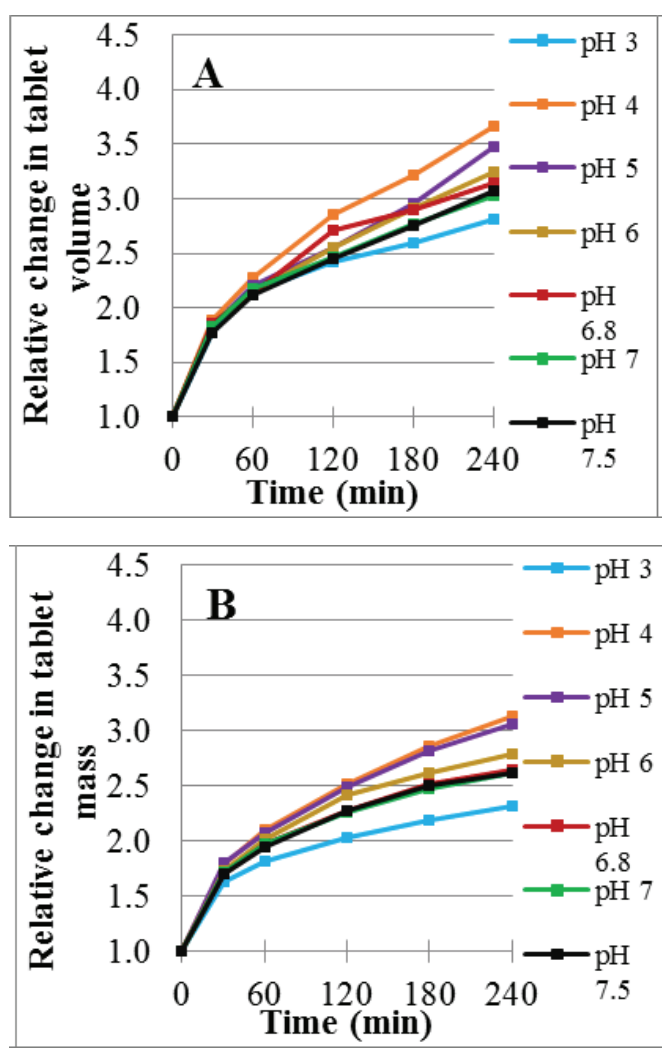

Figure 5. Relative swelling of tablets containing 50\% DF-Na in media with $\mathrm{pH}$ values from 3.0 to 7.5. (A) Swelling determined using cameras; (B) swelling determined by weighing.

Visualization of the swelling process may contribute to a clearer explanation. Photographs of tablets during dissolution are shown in Figure 6. Tablet size increased with time, although differences in size at different $\mathrm{pH}$ values were relatively small and in alignment with the swelling results shown in Figure 5. The swelled layer is clearly seen at higher $\mathrm{pH}$ values, whereas at $\mathrm{pH} 3.0$ (Figure 6), pH 4.0 (not shown), and pH 5.0 (Figure 6), tablets appear completely white and nontransparent. At these lower $\mathrm{pH}$ values, tablet size also increases with time. Photographs show only tablet size increases, and in media with lower pH values, the swelled layer itself cannot be distinguished from the dry matrix core because of its white, nontransparent appearance. Thus, diclofenac in the form of a sodium salt dissolves at lower $\mathrm{pH}$ values when in contact with an aqueous medium but precipitates in the form of acid immediately after exposure to low $\mathrm{pH}$. The layer of undissolved or precipitated diclofenac (acid form) is presumed to be on the surface of the tablets, thus preventing the swelled layer from being observed. In the present study, only the overall dimensions of the tablets were read from photographs, although the width of the swelled layer itself may also be determined by cameras (when it is clearly visible) as at higher pH values (Figure 6). 


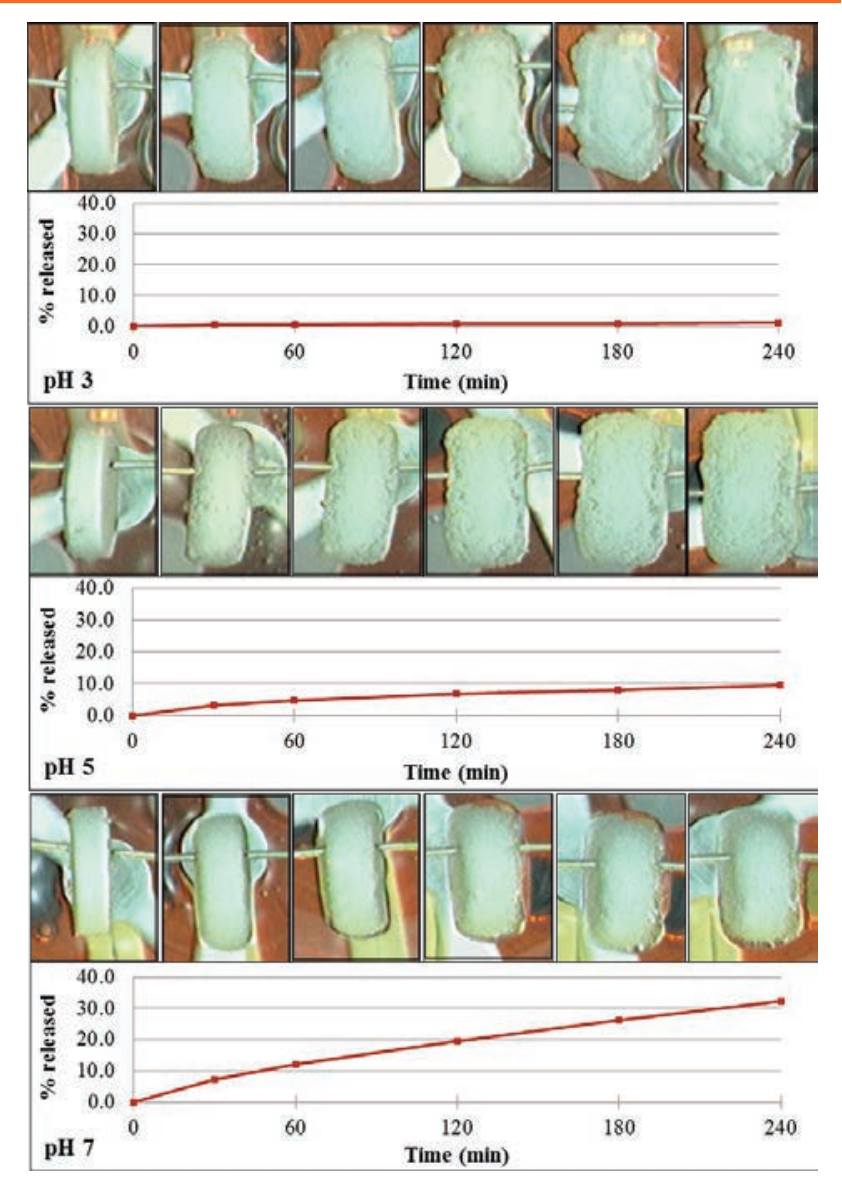

Figure 6. Dissolution profiles for tablets containing 50\% DF-Na in media at $\mathrm{pH} 3.0,5.0$, and 7.0. Images on the individual graph are of one tablet at sampling points $0,30,60,120,180$, and $240 \mathrm{~min}$. The diameter of the dry tablet was $1.2 \mathrm{~cm}$; thickness was $0.3 \mathrm{~cm}$.

As the $\mathrm{pH}$ value of the medium used is increased, its ionic strength also increases and might influence swelling and drug release from HPMC matrices (24-27). To gain additional insight into the role of physicochemical properties of the drug, especially its solubility and the influence of medium ionic strength, these processes were also followed for tablets containing paracetamol. The solubility of paracetamol was not expected to present a limitation regarding the volume of medium used at any tested $\mathrm{pH}$ value $\left(\mathrm{p} K_{\mathrm{a}}=9.5\right)(28)$. Dissolution profiles of tablets containing $50 \%$ paracetamol are shown in Figure 7 , and swelling of the same tablets, determined in both methods, is shown in Figure 8. As expected and in contrast to DF-Na tablets, drug release from paracetamol tablets was not $\mathrm{pH}$-dependent, and the swelling of paracetamol tablets was similar in media at all tested $\mathrm{pH}$ values. Some differences in swelling at different $\mathrm{pH}$ values were observed using the camera method and are probably the result of experimental errors, which are also reflected in the greater standard deviations (Figure 8A) than with the tablet weighing method (Figure 8B). Similar conclusions can be made for tablets containing a lower amount of paracetamol (25\%). Differences in ionic strength relating to the composition of the media at different $\mathrm{pH}$ values do not obviously affect swelling or dissolution.

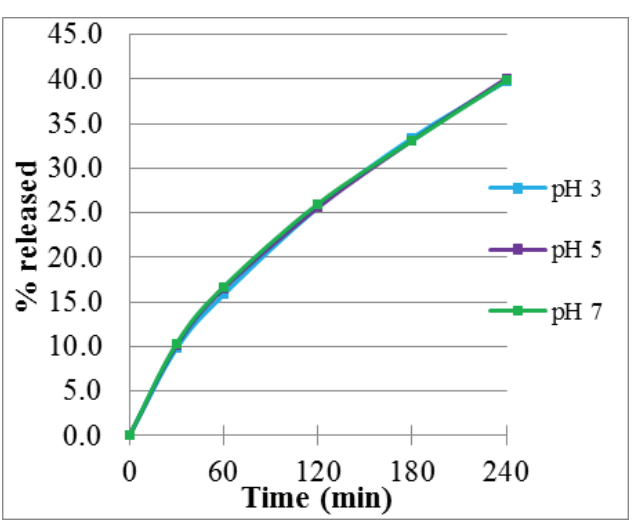

Figure 7. Drug release from tablets containing $50 \%$ paracetamol in media at $\mathrm{pH}$ 3.0, 5.0, and 7.0.
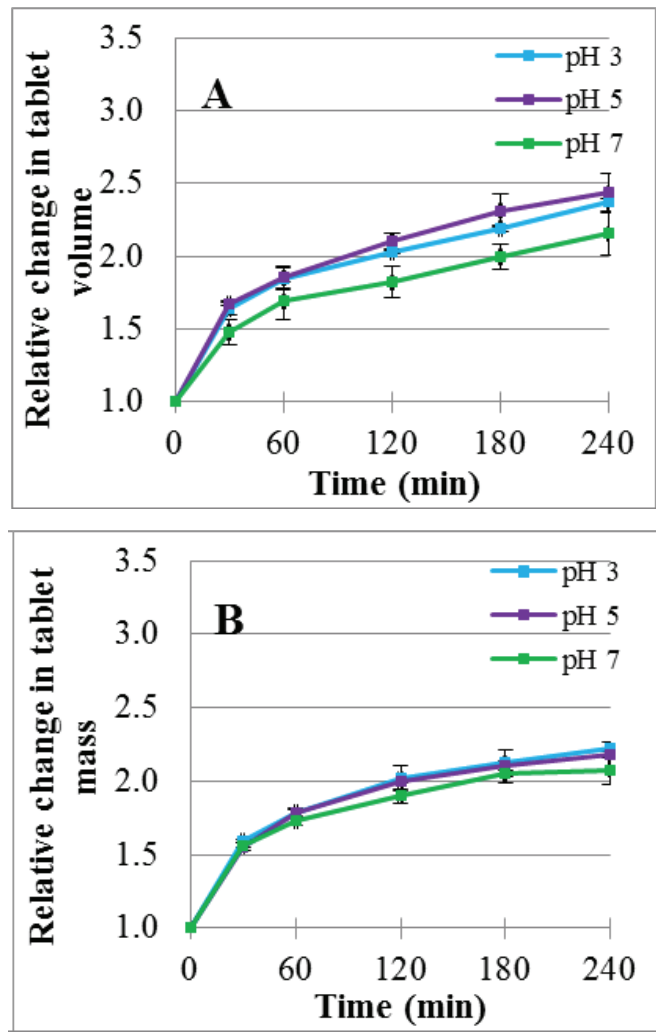

Figure 8. Relative swelling of tablets containing 50\% paracetamol in media at $\mathrm{pH}$ 3.0, 5.0, and 7.0: (A) swelling determined using cameras and $(B)$ swelling determined by weighing $(n=3$; values are mean $\pm S D$ ). 


\section{CONCLUSION}

The swelling behavior of matrix tablets was monitored using both a camera system placed underneath the dissolution apparatus and a weighing method. Using the camera system, dissolution and swelling were monitored simultaneously without interfering with the dissolution process. The correlation between swelling results obtained in the two methods was high at all $\mathrm{pH}$ values of the medium. Some difficulties were encountered when working with the camera system, such as the effect of light refraction, variation of tablet shape after time in the dissolution medium, and tablet position. Some problems were resolved by calculating only relative swelling values; other problems could be minimized by upgrading the measuring quality of the cameras and the corresponding software. Nevertheless, the bottom-view camera system used during dissolution testing provided additional information and insight into drug release and the swelling process of DF-Na/HPMC tablets.

\section{ACKNOWLEDGMENT}

The authors thank Merel d.o.o., Slovenia, for enabling us to work with the dissoGUARD PRO dissolution surveillance system.

\section{CONFLICT OF INTEREST}

The authors declare that there is no conflict of interest.

\section{REFERENCES}

1. Kazarian, S. G.; van der Weerd, J. Simultaneous FTIR Spectroscopic Imaging and Visible Photography to Monitor Tablet Dissolution and Drug Release. Pharm. Res. 2008, 25 (4), 853-860. DOI: 10.1007/s11095007-9375-4.

2. Morita, Y.; Tsushima, Y.; Yasui, M.; Termoz, R.; Ajioka, J.; Takayama, K. Evaluation of the Disintegration Time of Rapidly Disintegrating Tablets via a Novel Method Utilizing a CCD Camera. Chem. Pharm. Bull. 2002, 50 (9), 1181-1186. DOI: 10.1248/cpb.50.1181.

3. Tres, F.; Treacher, K.; Booth, J.; Hughes, L. P.; Wren, S. A.; Aylott, J. W.; Burley, J. C. Real time Raman imaging to understand dissolution performance of amorphous solid dispersions. J. Controlled Release 2014, 188, 5360. DOI: 10.1016/j.jconrel.2014.05.061.

4. Cao, Q.-R.; Choi, H.-G.; Kim, D.-C.; Lee, B.J. Release behavior and photo-image of nifedipine tablet coated with high viscosity grade hydroxypropylmethylcellulose: effect of coating conditions. Int. J. Pharm. 2004, 274 (1-2), 107-117. DOI: 10.1016/j.ijpharm.2004.01.020.

5. Tieu, K.; Salt, A.; Wirges, J.; Grove, G. N. Regulatory Considerations for the Classification of Video Monitoring in Dissolution Testing. AAPS PharmSciTech
2014, 15 (6), 1611-1618. DOI: 10.1208/s12249-0140191-y.

6. Ford, J. L. Design and Evaluation of Hydroxypropyl Methylcellulose Matrix Tablets for Oral Controlled Release: A Historical Perspective. In Hydrophilic Matrix Tablets for Oral Controlled Release; Timmins, P., Pygall, S., Melia, C. D., Eds.; AAPS Advances in the Pharmaceutical Sciences, Vol. 16; Springer: New York, 2014; pp 17-51. DOI: 10.1007/978-1-4939-151942 .

7. Gao, P.; Skoug, J. W.; Nixon, P. R.; Ju, T. R.; Stemm, N. L.; Sung, K. C. Swelling of Hydroxypropyl Methylcellulose Matrix Tablets. 2. Mechanistic Study of the Influence of Formulation Variables on Matrix Performance and Drug Release. J. Pharm. Sci. 1996, 85 (7), 732-740. DOI: 10.1021/js9504595.

8. Colombo, P.; Bettini, R.; Santi, P.; Peppas, N. A. Swellable matrices for controlled drug delivery: gel-layer behaviour, mechanisms and optimal performance. Pharm. Sci. Technol. Today 2000, 3 (6), 198-204. DOI: 10.1016/S1461-5347(00)00269-8.

9. Kiil, S.; Dam-Johansen, K. Controlled drug delivery from swellable hydroxypropylmethylcellulose matrices: model-based analysis of observed radial front movements. J. Controlled Release 2003, 90 (1), 1-21. DOI: 10.1016/S0168-3659(03)00122-6.

10. Pham, A. T.; Lee, P. I. Probing the Mechanisms of Drug Release from Hydroxypropylmethyl Cellulose Matrices. Pharm. Res. 1994, 11 (10), 1379-1384. DOI: 10.1023/A:1018975318805.

11. Colombo, P.; Bettini, R.; Santi, P.; De Ascentiis, A.; Peppas, N. A. Analysis of the swelling and release mechanisms from drug delivery systems with emphasis on drug solubility and water transport. J. Controlled Release 1996, 39 (2-3), 231-237. DOI: 10.1016/0168-3659(95)00158-1.

12. Tahara, K.; Yamamoto, K.; Nishihata, T. Application of model-independent and model analysis for the investigation of effect of drug solubility on its release rate from hydroxypropyl methylcellulose sustained release tablets. Int. J. Pharm. 1996, 133 (1-2), 17-27. DOI: 10.1016/0378-5173(95)04400-0.

13. Caraballo, I. Factors affecting drug release from hydroxypropyl methylcellulose matrix systems in the light of classical and percolation theories. Expert Opin. Drug Delivery 2010, 7 (11), 1291-1301. DOI: 10.1517/17425247.2010.528199.

14. Bettini, R.; Catellani, P. L.; Santi, P.; Massimo, G.; Peppas, N. A.; Colombo, P. Translocation of drug particles in HPMC matrix gel layer: effect of drug solubility and influence on release rate. J. Controlled Release 2001, 70 (3), 383-391. DOI: 10.1016/ 
S0168-3659(00)00366-7.

15. Yang, L.; Fassihi, R. Examination of drug solubility, polymer types, hydrodynamics and loading dose on drug release behavior from a triple-layer asymmetric configuration delivery system. Int. J. Pharm. 1997, 155 (2), 219-229. DOI: 10.1016/S0378-5173(97)00164-6.

16. Li, C. L.; Martini, L. G.; Ford, J. L.; Roberts, M. The use of hypromellose in oral drug delivery. $J$. Pharm. Pharmacol. 2005, 57 (5), 533-546. DOI: 10.1211/0022357055957.

17. dissoGUARD Dissolution Surveillance System. Merel d.o.o. Web site. http://www.dissoguard.com (accessed Oct 17, 2016).

18. Rajabi-Siahboomi, A. R.; Bowtell, R. W.; Mansfield, P.; Henderson, A.; Davies, M. C.; Melia, C. D. Structure and behaviour in hydrophilic matrix sustained release dosage forms: 2. NMR-imaging studies of dimensional changes in the gel layer and core of HPMC tablets undergoing hydration. J. Controlled Release 1994, 31 (2), 121-128. DOI: 10.1016/0168-3659(94)00016-6.

19. Chen, Y.-C.; Ho, H.-O.; Liu, D.-Z.; Siow, W.-S.; Sheu, M.-T. Swelling/Floating Capability and Drug Release Characterizations of Gastroretentive Drug Delivery System Based on a Combination of Hydroxyethyl Cellulose and Sodium Carboxymethyl Cellulose. PLOS ONE 2015, 10 (1), e0116914. DOI: 10.1371/journal. pone. 0116914 .

20. Kavanagh, N.; Corrigan, O. I. Swelling and erosion properties of hydroxypropylmethylcellulose (Hypromellose) matrices-influence of agitation rate and dissolution medium composition. Int. J. Pharm. 2004, 279 (1-2), 141-152. DOI: 10.1016/j. ijpharm.2004.04.016.

21. Nagelj Kovačič, N.; Pišlar, M.; llić, I.; Mrhar, A.; Bogataj, $M$. Influence of the physiological variability of fasted gastric $\mathrm{pH}$ and tablet retention time on the variability of in vitro dissolution and simulated plasma profiles. Int. J. Pharm. 2014, 473 (1-2), 552-559. DOI: 10.1016/j.ijpharm.2014.07.031.

22. Kincl, M.; Meleh, M.; Veber, M.; Vrečer, F. Study of physicochemical parameters affecting the release of diclofenac sodium from lipophilic matrix tablets. Acta Chim. Slov. 2004, 51 (3), 409-425.

23. Chiarini, A.; Tartarini, A.; Fini, A. pH-Solubility Relationship and Partition Coefficients for some AntiInflammatory Arylaliphatic Acids. Arch. Pharm. 1984, 317 (3), 268-273. DOI: 10.1002/ardp.19843170314.

24. Asare-Addo, K.; Levina, M.; Rajabi-Siahboomi, A. R.; Nokhodchi, A. Effect of ionic strength and $\mathrm{pH}$ of dissolution media on theophylline release from hypromellose matrix tablets-Apparatus USP III, simulated fasted and fed conditions. Carbohydr. Polym. 2011, 86 (1), 85-93. DOI: 10.1016/j. carbpol.2011.04.014.

25. Joshi, S. C. Sol-Gel Behavior of Hydroxypropyl Methylcellulose (HPMC) in lonic Media Including Drug Release. Materials 2011, 4 (10), 1861-1905. DOI: 10.3390/ma4101861.

26. Xu, X. M.; Song, Y. M.; Ping, Q. N.; Wang, Y.; Liu, X. Y. Effect of ionic strength on the temperature-dependent behavior of hydroxypropyl methylcellulose solution and matrix tablet. J. Appl. Polym. Sci. 2006, 102 (4), 4066-4074. DOI: 10.1002/app.24393.

27. Krese, A.; Nagelj Kovačič, N.; Kapele, T.; Mrhar, A.; Bogataj, M. Influence of ionic strength and HPMC viscosity grade on drug release and swelling behavior of HPMC matrix tablets. J. Appl. Polym. Sci. 2016, 133 (26), 43604. DOI: 10.1002/APP.43604.

28. Reynolds, J. E. F., Prasad, A. B., Eds. Martindale: The Extra Pharmacopoeia, 28th ed.; Pharmaceutical Press: London, 1982; pp xxvi. 